\title{
Estrategias para el trabajo tutorial en la Facultad de Pedagogía, Universidad Veracruzana
}

\section{Resumen}

$\mathrm{S}$ e muestran las líneas de acción empleadas por algunos docentes de la Facultad de Pedagogía de la Universidad Veracruzana que desempeñan la función de tutor académico. Las acciones tienen la finalidad de contribuir al desarrollo de la formación integral de los estudiantes, propósito establecido en el Modelo Educativo Institucional de la universidad. El artículo se fundamenta en los documentos rectores institucionales y en las prácticas emprendidas por los tutores académicos, el cual tiene como objetivo brindar acompañamiento a los estudiantes a lo largo de su trayectoria académica, mediante la atención personalizada o grupal, durante el proceso de ingreso, permanencia y egreso; tomando como referencia los intereses, motivaciones, expectativas y necesidades de los estudiantes, así como el perfil de egreso, para consolidar las competencias profesionales, en el marco de la formación integral y bajo el impulso de los temas transversales emprendidos por la institución.

Palabras clave: trabajo tutorial, acompañamiento, trayectoria académica, formación integral

\section{Introducción}

La Universidad Veracruzana (Uv) reconoce el trabajo tutorial desde 1999, plasmado en documentos institucionales de suma importancia, como son el nuevo Modelo Educativo para la Universidad Veracruzana, el Reglamento del Sistema Institucional de Tutorías, el Estatuto de Personal Académico, el Reglamento del Programa de Estímulos al Desempeño del Personal Académico, los Lineamientos de operación para Enseñanza Tutorial: Tutoría para la Investigación, Tutoría para la Apreciación Artística y el Programa de Monitores.

A partir del año 1999, con la implementación del Nuevo Modelo Educativo para la Uni- versidad Veracruzana ${ }^{1}$, se comienza a hablar de la tutoría como una estrategia de trabajo académico que permita contribuir a la formación integral de los estudiantes, tomando como referencia los cuatro ámbitos: intelectual, humano, social y profesional. En el tenor de la flexibilidad, se demanda la responsabilidad y la autonomía del alumnado para la toma de decisiones sobre su carga crediticia. En este sentido, emerge el trabajo tutorial como "una herramienta fundamental para contribuir en el logro de tales fines y brindar la información y el apoyo necesario a los alumnos en la toma de decisiones académicas" (Universidad Veracruzana, 2009, p. 3).

1 Nuevo Modelo Educativo para la Universidad Veracruzana. Disponible en: https://www.uv.mx/meif/files/2015/03/MEIF.pdf. 
Al respecto, la actividad tutorial demanda un tutor integral que tenga la capacidad de guiar a los estudiantes, favoreciendo la adecuada toma de decisiones de manera oportuna, así como brindar herramientas que permitan atender el ámbito académico, encaminado al desarrollo de competencias indispensables para el aprendizaje significativo (Beltrán y Suárez, 2003).

Institucionalmente se hace énfasis en las modalidades de tutoría académica y enseñanza tutorial. La tutoría académica está enfocada en atender diversas situaciones de los estudiantes en donde requieren información, especialmente relacionada con su plan de estudios, la seriación y elección de experiencias educativas (EE), el total de los créditos a cursar por semestre y todo lo que conlleva la trayectoria escolar; asimismo, se pretende hacer énfasis en los valores y las actitudes inherentes a la formación, y que vayan desarrollando una cultura de autoaprendizaje. Por su parte, la enseñanza tutorial hace referencia al apoyo hacia el estudiante en situaciones problemáticas que enfrenta en su disciplina, con relación al contenido y a las habilidades para el aprendizaje de la misma. Se busca intervenir cuando: los estudiantes no comprenden algún contenido o tengan interés de profundizar en algún conocimiento particular (Beltrán y Suárez, 2003; Universidad Veracruzana, 2009).

Por consiguiente, el papel del tutor se centra en diversas funciones, en las que se destaca: formar, informar, prevenir y ayudar a decidir. $\mathrm{La}$ primera permite crear las condiciones para el desarrollo de competencias encaminadas a la formación integral. La segunda ayuda a dar a conocer a los demás docentes y estudiantes sobre lo que acontece en la realidad institucional relacionada con los tutorados, así como brindar información sobre las propuestas educativas, culturales, deportivas $\mathrm{y} / \mathrm{o}$ artísticas que oferta la universidad. La tercera consiste en estar pendiente de las situaciones posibles de riesgo que limiten la formación, así como prever con acciones inmediatas. La última está llamada a brindar orientación para que los estudiantes puedan tomar decisiones relacionadas con su perfil profesional, desde su ingreso hasta su egreso. Sin duda, estas funciones están orientadas a integrar un diagnóstico individual de los tutorados y a dar seguimiento durante la trayectoria escolar (González y Solano, 2015; Beltrán y Suárez, 2003).
Al margen de lo anterior, es importante resaltar las cualidades que como tutores académicos se hacen presentes, en las que podemos destacar: "inspirar confianza, saber ayudar delicada y desinteresadamente, estar disponible, no manifestar preferencia" (Mañú, 2016, p. 25). De igual manera, podemos enfatizar en apoyar a los estudiantes cuando lo requieran, a través de la participación del tutor, estableciendo canales de comunicación, brindar información relacionada con el plan de estudios, campo profesional y laboral, y los lineamientos académico-administrativos (Pérez, García y Guerrero, 2012). Aunado a ello, la uv plantea el perfil general del tutor, donde se pueden apreciar otras funciones, como "acreditar el conocimiento: del Nuevo Modelo Educativo, de los aspectos conceptuales y prácticos del ejercicio de las tutorías, identificarse con la misión y la filosofía de la universidad y contar con experiencia docente", entre otras (Beltrán, 2000, p. 16).

\section{Desarrollo}

La Coordinación del Sistema Tutorial tiene la responsabilidad de planear, organizar, ejecutar, dar seguimiento y evaluar la actividad tutorial. Como parte de las acciones institucionales, el proceso de tutoría se lleva a cabo de manera formal, considerando el mínimo de tres sesiones en el periodo semestral; sin embargo, es posible realizar más sesiones, en virtud de la disponibilidad del tutor y a solicitud de los tutorados (Beltrán y Suárez, 2003). Cabe destacar que las fechas para llevarse a cabo y los temas administrativos son indicados por la coordinación.

Como primer punto, al formar parte de los tutores académicos, se debe elaborar un plan de trabajo que permita integrar una propuesta de acciones a realizar durante el año, que dé muestra del acompañamiento que se les brindará a los estudiantes y permita brindar un seguimiento a su trayectoria académica en los tres momentos tutoriales.

En el primer momento tutorial es preciso tener acercamiento con los tutorados, en el que se pueda conocer a quiénes se les brindará acompañamiento, conociendo más de ellos; para esto, es necesario aplicar diversas técnicas e instrumentos de diagnóstico a los estudiantes, empezando por 
conocerlos como personas, sus intereses, motivaciones, expectativas, áreas de oportunidad y fortalezas -tanto académicas como personales-, entre otras. Una de las acciones inherentes a este primer momento tutorial es brindarles información del plan de estudios al que están inscritos, su estructura, darles a conocer sus objetivos curriculares, competencias del perfil de egreso, el mapa curricular y otros elementos que el tutor considere pertinentes en ese momento. Asimismo, no se puede dejar de lado dar a conocer a los estudiantes el proceso de preinscripción e inscripción en línea; proporcionarles información de los servicios que brinda la universidad y la facultad, además de los programas institucionales en los que pueden incorporarse; informarles los procesos administrativos que deben realizar en su proceso de ingreso; invitarlos a utilizar el correo institucional como medio importante para conocer los eventos académicos, culturales, artísticos y deportivos; revisar continuamente la página web oficial de la universidad y de la facultad de pedagogía; así como brindarles información sobre el examen de competencias de inglés y de las becas. En este sentido, se considera valioso establecer medios de comunicación, por lo que se abren grupos de WhatsApp y Facebook para estar en contacto continuamente.

Durante el segundo momento tutorial es importante tener presente el seguimiento a la trayectoria de los estudiantes a través de la revisión del avance crediticio, prestando atención a la carga mínima, que corresponde a la inscripción de 32 créditos por periodo en doce semestres; la carga estándar de 48 créditos que permite el egreso en ocho semestres; y la carga máxima, correspondiente a 64 créditos para concluir en seis semestres. Asimismo, es importante monitorear el avance que presentan los estudiantes y sus calificaciones a través del Sistema Institucional de Tutorías (SIT), ya que éstas son señales que nos permiten atender a través de diversas acciones las áreas de oportunidad de algunas experiencias educativas en las que los estudiantes necesiten reforzamiento.

Otro de los aspectos del quehacer tutorial es la atención constante a las dudas de los estudiantes en torno a su situación académica o administrativa y, en ocasiones, de índole personal; debido a que surgen con regularidad y de manera emergente, por lo que deben atenderse inmediatamente, o bien, canalizarse con los especialistas en el tema. Es importante que durante este proceso se realice un diagnóstico que permita atender a los estudiantes en situaciones de riesgo, dado que, si no hay una detección oportuna, éstos pueden desertar de la carrera.

El tercer momento se caracteriza por ofrecer seguimiento a la trayectoria de los estudiantes a través de la revisión del avance crediticio, acción que se viene desempeñando desde el momento anterior; atender las dudas en torno a situaciones académicas, administrativas y personales; la atención de los estudiantes en situación de riesgo; la difusión de información sobre las fechas de exámenes ordinarios de las experiencias educativas, e invitar a los estudiantes a realizar la evaluación de los docentes y del tutor.

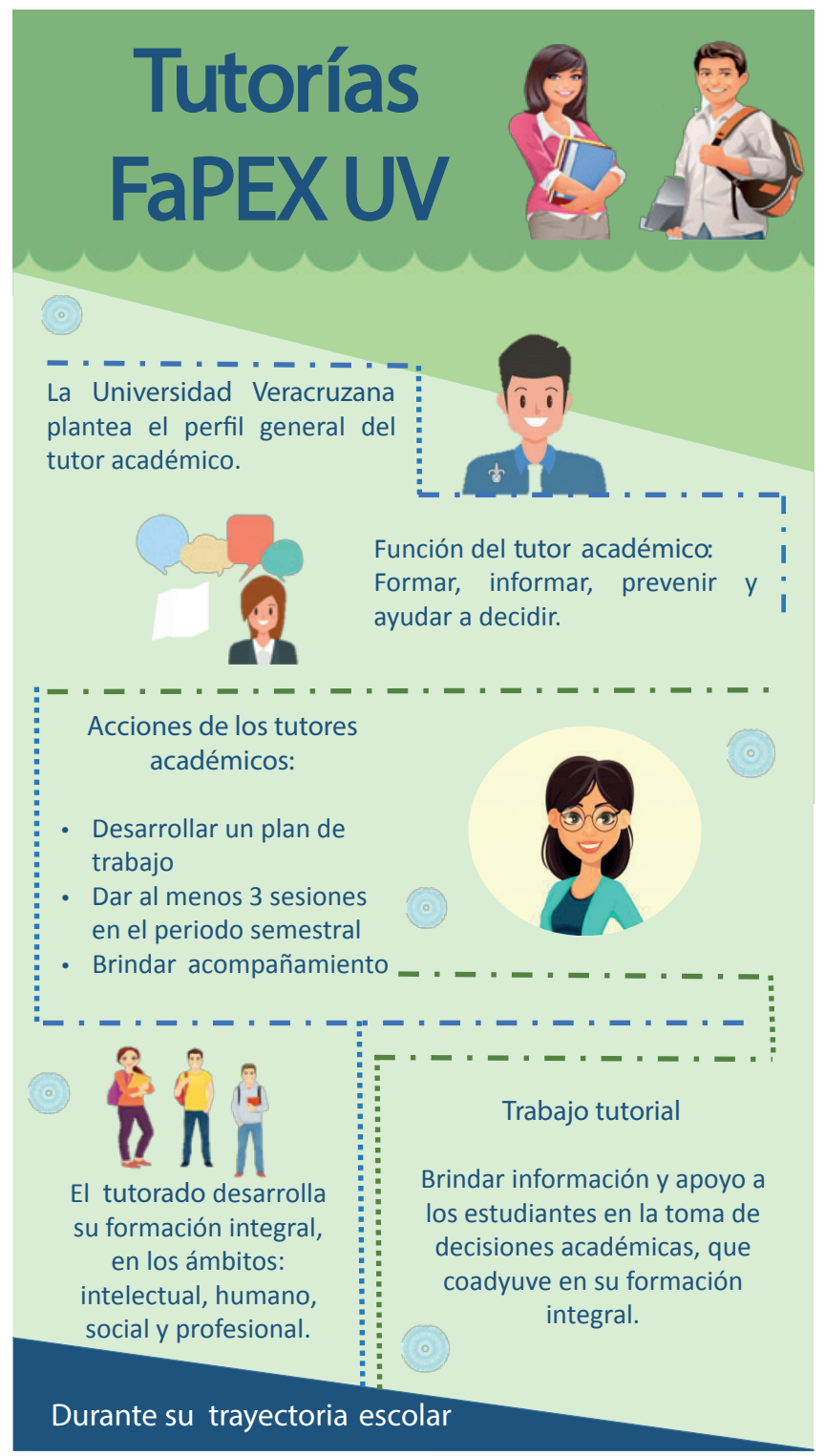




\section{Conclusiones}

Es preciso que los tutores académicos posean estrategias para orientar la atención y el seguimiento de los estudiantes, entre las que destacan: el diseño de acciones que potencien los indicadores de aprobación y eficiencia terminal, a través de la asesoría académica personalizada en temas que requieran atención, o bien, la asesoría académica grupal en temas comunes. Asimismo, es importante la implementación de acciones para la detección y atención a estudiantes en riesgo académico, como la estrategia de compañero-guía, en el cual otro alumno que posee la información pueda ayudar a su compañero, de par a par, en los temas que requiere.

A partir de lo antes establecido sobre las necesidades en la función tutorial, se considera oportuna la coordinación de reuniones de trabajo con los tutores interesados, con el propósito de detectar qué estudiantes se encuentran en riesgo; así, de manera colaborativa, establecer líneas de acción en beneficio de su formación, como la elaboración de un reporte mensual sobre su situación y el diseño, tanto de planes de nivelación como de materiales de apoyo a disposición de los mismos, entre otros.

Por último, es necesario establecer en un calendario institucional permanente las acciones orientadas a fomentar la participación de los estudiantes en actividades de integración a la vida universitaria, el acceso a los servicios, el cuidado de la sana convivencia estudiantil y de la salud emo- cional, entre otras; el cual se promueva a través de redes sociales y otros materiales de difusión para hacer conciencia sobre su importancia. Sin duda, la naturaleza del quehacer tutorial de la Universidad Veracruzana busca atender la formación integral de los estudiantes que contribuya al logro del perfil de competencias y al desarrollo personal y profesional.

\section{Fuentes de consulta}

Beltrán, J. (2000). Guía para el ejercicio de la tutoría. Xalapa: Universidad Veracruzana.

Beltrán, J. y Suárez, J. (2003). El quehacer tutorial. Guía de trabajo. Xalapa: Universidad Veracruzana.

González, A. y Solano, J. (2015). La función de tutoría. Carta de navegación para tutores. Madrid: Narcea Ediciones.

Mañú, J. (2016). Manual de tutorías. Madrid: Narcea Ediciones.

Pérez, R., García, G. y Guerrero, K. (2012). El sistema tutorial: percepción de tutores, tutorados y coordinadores del ST. Xalapa: Universidad Veracruzana.

Universidad Veracruzana (29/jun/2009). Legislación Universitaria. Reglamento del sistema institucional de tutorías. Aprobado en sesión del H. Consejo Universitario General. Xalapa: uv. 